\title{
Prognostic and clinicopathological value of ZIC1 in patients with cervical squamous cell carcinoma
}

\author{
$\mathrm{XING} \mathrm{GU}^{1 *}$, XUE-KE GUO ${ }^{2 *}$, BI-HUI CHEN ${ }^{3 *}$, XIAO-JIAO GAO ${ }^{4}$, FANG CHEN $^{4}$ and QIN LIU $^{1}$ \\ ${ }^{1}$ Department of Gynecology, Kunshan First People's Hospital Affiliated to Jiangsu University, Kunshan, Jiangsu 215300; \\ ${ }^{2}$ Department of Gynecology, The Third Affiliated Hospital of Wenzhou Medical University, Wenzhou, Zhejiang 325000; \\ ${ }^{3}$ Department of Gynecology, Kunshan Hospital of Traditional Chinese Medicine; ${ }^{4}$ Department of Pathology, Kunshan \\ First People's Hospital Affiliated to Jiangsu University, Kunshan, Jiangsu 215300, P.R. China
}

Received April 10, 2019; Accepted August 30, 2019

DOI: $10.3892 / \mathrm{ol} .2019 .11007$

\begin{abstract}
The aim of the present study was to analyze the differences in zinc finger of the cerebellum 1 (ZIC1) expression between cervical cancer tissue, precancer tissue and normal cervical tissue to determine its clinicopathological and prognostic value in cervical squamous cell carcinoma (CSCC). Reverse transcription-quantitative PCR was used to determine the mRNA expression levels of ZIC1 in 569 fresh-frozen biopsy tissues, and immunohistochemistry was performed to detect ZIC1 protein expression in $80 \mathrm{CSCC}$ tissues and 320 cervical intraepithelial neoplasia (CIN) grade III samples. The association of ZIC1 expression with the clinicopathological characteristics of CSCC was then analyzed using Cox regression analysis, and Kaplan-Meier curves were used to analyze the prognostic value. The level of ZIC1 mRNA expression in CSCC was significantly lower compared with normal cervical tissues and CIN I-III tissues $(\mathrm{P}<0.001)$. There was a negative correlation between ZIC1 immunoreactivity score (IRS) in CSCC tissue and adjacent noncancerous tissue $(\mathrm{R}=-0.279 ; \mathrm{P}=0.012)$; the mean IRS of $\mathrm{ZIC} 1$ in CSCC tissue
\end{abstract}

Correspondence to: Professor Qin Liu, Department of Gynecology, Kunshan First People's Hospital Affiliated to Jiangsu University, 5 Qingyang Mid Road, Kunshan, Jiangsu 215300, P.R. China E-mail: 1073938215@qq.com

*Contributed equally

Abbreviations: HPV, human papillomavirus; CC, cervical cancer; CSCC, cervical squamous cell carcinoma; CIN, cervical intraepithelial neoplasia; ZIC, zinc finger of the cerebellum; IHC, immunohistochemistry; IRS, immunoreactivity score; OS, overall survival; DFS, disease-free survival; RT-qPCR, reverse transcription-quantitative PCR; Leep, loop electrosurgical excision procedure; FIGO, International Federation of Gynecology and Obstetrics

Key words: zinc finger of the cerebellum 1, cervical squamous cell carcinoma, prognosis, clinicopathological features, cervical intraepithelial neoplasia was $5.36 \pm 3.48$, which was significantly lower compared with the corresponding adjacent noncancerous tissues $(11.31 \pm 5.68$; $\mathrm{P}<0.001)$ and CIN III samples $(10.42 \pm 1.54$; $\mathrm{P}<0.001)$. In addition, expression of ZIC1 was negatively associated with International Federation of Gynecology and Obstetrics (FIGO) stage $(\mathrm{P}=0.027)$ and lymph node metastasis $(\mathrm{P}<0.001)$. In $\mathrm{Cox}$ regression analysis, $\mathrm{ZIC1}$ expression [hazard ratio (HR), 0.61; 95\% confidence interval (CI), 0.40-0.92; $\mathrm{P}=0.018$ ), FIGO staging ( HR, 3.55; 95\% CI, 2.35-5.37; $\mathrm{P}<0.001)$ and lymph node metastasis (HR, 2.50; 95\% CI, 1.62-3.86; $\mathrm{P}<0.001)$ were three independent prognostic factors of overall survival. Furthermore, ZIC1 expression was also associated with disease-free survival $(\mathrm{P}=0.003)$. These results suggest that ZIC1 expression in CSCC may be lower than in normal cervical tissues or CIN tissues, and high expression of ZIC1 may be negatively associated with FIGO stage and lymph node metastasis. Therefore, ZIC1 may be a promising biomarker for the prognosis of CSCC.

\section{Introduction}

Human papillomavirus (HPV) infection is the primary cause of the majority of cases of cervical cancer (CC) worldwide, and $\mathrm{CC}$ has become one of the leading causes of cancer-associated death among females in 2018 (1). The most common type of CC is cervical squamous cell carcinoma (CSCC) (1). The prognosis of cervical cancer remains poor as it has a high rate of metastasis and recurrence (2). Cervical intraepithelial neoplasia (CIN) are types of precancerous lesions closely associated with cervical carcinoma, including CIN I-III which reflect the continuous development of cervical cancer (3). Therefore, it is crucial to identify novel prognostic markers for auxiliary assessment of CIN grading and prognosis of patients with CSCC.

Zinc finger of the cerebellum 1 (ZIC1) was first identified in cerebellum tissues in 1994 (4). A previous study has shown that ZIC1 serves an important role in the growth and development of the brain nervous system, muscle cell production and bone development (5). In addition, methylation of $\mathrm{ZIC1}$ is associated with the development and progression of cervical cancer (6). Ectopic ZIC1 expression was found to suppress the growth of colorectal cancer cells by reducing phosphorylation of Akt and 
Erk (7). Furthermore, ZIC1 downregulation has been associated with metastasis and a poor prognosis in patients with breast carcinoma or gastric cancer $(8,9)$. However, a previous study reported that ZIC1 was upregulated in endometrial cancer in Hong Kong Chinese women (10). Our previous study also revealed that $\mathrm{ZIC1}$ was upregulated in endometrial cancer compared with corresponding normal tissue, and was positively correlated with endometrial carcinogenesis (11). Although ZIC1 exhibits different functions in neoplasms compared with normal physiological tissue, $\mathrm{ZIC1}$ is thought to be essential for carcinogenesis, and may thus serve as a novel target for treating patients with cervical cancer $(10,11)$.

Based on the reported effects of $\mathrm{ZIC1}$ on cervical carcinoma, the aim of the present study was to determine the mRNA expression levels of ZIC1 in normal cervical tissue, CIN samples and CSCC tissues by using reverse transcription-quantitative PCR (RT-qPCR), and to compare $\mathrm{ZIC1}$ expression between the three tissue types. In addition, the protein expression levels of ZIC1 in 80 cases of CSCC with radical hysterectomy were determined using immunohistochemistry (IHC) to examine the clinicopathological and prognostic value of ZIC1 in patients with CSCC.

\section{Materials and methods}

Tissue samples. The present study was approved by The Ethics Committee of Kunshan First People's Hospital (Jiangsu, China), and written informed consent was obtained from each patient for the use of their tissues for research and publication. A total of 569 fresh-frozen biopsy tissues comprised of normal cervical tissues $(n=400)$, CIN I $(n=50)$, CIN II $(n=36)$, CIN III $(n=38)$ and CSCC $(n=45)$ were obtained from Kunshan First People's Hospital and the mRNA was extracted. These patients were recruited between January 2010 and December 2018, and the mean age of the patients was $53.26 \pm 11.08$ years. Additionally, 80 patients (the mean age was $57.39 \pm 8.64$ years) with CSCC at International Federation of Gynecology and Obstetrics (FIGO) stage IA, IB or IIA underwent radical hysterectomy at Kunshan First People's Hospital between January 2009 and January 2013 (12). The patients included in the study had not received chemotherapy or radiotherapy prior to surgery. The tumor specimens (mean size, $2.73 \pm 1.06 \mathrm{~cm}$ ) and the corresponding adjacent noncancerous tissues were fixed in $10 \%$ formalin at $20^{\circ} \mathrm{C}$ for $8 \mathrm{~h}$ and then embedded in paraffin blocks. Complete clinical and pathological data were available for all the patients. All patients received carboplatin + paclitaxel treatment regimen following surgery. The mean age of the patients was $53.10 \pm 12.76$ years, and the follow-up duration ranged from 3-60 months, with a mean follow-up duration of $46.313 \pm 2.317$ months. In addition, the pathological tissues of 320 patients with CIN III (the mean age was 47.21 \pm 9.07 years and recruited between January 2010 and December 2018) who underwent a loop electrosurgical excision procedure (Leep) at Kunshan First People's Hospital were also stored in paraffin blocks for IHC analysis. The 569 cohort of patients, the 80 patients with CSCC and the 320 patients with CIN III were all proven to exhibit high-risk HPV infection using the Digene Hybrid Capture (r) 2 (HC2) High-Risk HPV DNA Test (Qiagen $\mathrm{GmbH}$ ), as previously described (13).
$R T-q P C R$. After pathological confirmation, total RNA was extracted using TRIzol ${ }^{\circledR}$ reagent (Thermo Fisher Scientific, Inc.), and $2 \mu \mathrm{g}$ RNA was reverse transcribed using the miScript II RT kit (Thermo Fisher Scientific, Inc.) at $50^{\circ} \mathrm{C}$ for $15 \mathrm{~min}$ then $85^{\circ} \mathrm{C}$ for 2 min. qPCR was performed using an iQ5 real-time PCR detection system (Bio-Rad Laboratories, Inc.) using SYBR Premix Ex Taq ${ }^{\mathrm{TM}}$ kit (Takara Bio, Inc.). The sequences of the primers were as follows: ZIC1 forward, 5'-GCGTCCTTTTGTGGATCTTTAA-3' and reverse, 5'-AGT AATCACATCTGCTTCTGGG-3'; and GAPDH forward, 5'-GAAGGTGAAGGTCGGAGT-3' and reverse, 5'-GAAGAT GGTGATGGGATTTC-3'. The PCR thermocycling conditions consisted of $94^{\circ} \mathrm{C}$ for $4 \mathrm{~min}$; followed by 40 cycles of $95^{\circ} \mathrm{C}$ for $1 \mathrm{~min}, 60^{\circ} \mathrm{C}$ for $1 \mathrm{~min}$ and $72^{\circ} \mathrm{C}$ for $1 \mathrm{~min}$. The relative mRNA expression levels were calculated using the $2^{-\Delta \Delta \mathrm{Cq}}$ method and the formula of $\Delta \Delta \mathrm{Cq}$ was $\left(\mathrm{Cq}_{\text {Tumor ZICl }}-\mathrm{Cq}_{\text {Tumor GAPDH }}\right)-\left(\mathrm{Cq}_{\text {Normal }}\right.$ $\left.\mathrm{ZICl}^{-} \mathrm{Cq}_{\text {Normal GAPDH }}\right)(14)$.

Hematoxylin and eosin staining. After deparaffinization and rehydration, $5 \mu \mathrm{m}$ longitudinal sections were stained with hematoxylin solution at $20^{\circ} \mathrm{C}$ for $5 \mathrm{~min}$ followed by 5 dips in $1 \%$ acid ethanol and then rinsed in distilled water. Sections were stained with eosin solution at $20^{\circ} \mathrm{C}$ for $3 \mathrm{~min}$, followed by dehydration with graded alcohol and clearing in xylene. The mounted slides were then examined and photographed using an inverted light microscope (magnification x40, 100 and 400; Nikon Corporation).

IHC and evaluation of immunohistochemical staining. Immunostaining for ZIC1 was performed using a SP rabbit and mouse horseradish peroxidase (HRP) kit (CoWin Biosciences) on tissue sections cut from formalin-fixed paraffin-embedded CSCC tumor lesions or CIN III samples. The ZIC1 rabbit polyclonal antibody (1:200; cat. no. bs-11609R; BIOSS) was used as the primary antibody. In addition, PBS without primary antibodies was used as a negative control. The samples were incubated with the primary antibody at $4^{\circ} \mathrm{C}$ overnight and subsequently, the biotinylated HRP secondary antibody $\left(1: 1,000\right.$; incubated at $20^{\circ} \mathrm{C}$ for $\left.15 \mathrm{~min}\right)$ and streptavidin-HRP conjugates (CoWin Biosciences) were used to detect ZIC1 expression. An immunoreactivity score (IRS) was calculated using a semi-quantitative assessment system for each case by two pathologists. The semi-quantitative assessment system was obtained by combining a score for staining intensity with a score for the staining percentage. The staining intensity score was defined as: 0 , no staining; 1 , mild staining; 2 , moderate staining; and 3, strong staining. The staining percentage score was defined as: $0,0 \% ; 1,1-10 \% ; 2,11-20 \% ; 3,21-30 \%$; 4, 31-40\%; 5, 41-50\%; 6, 51-60\%; 7, 61-70\%; 8, 71-80\%; 9 , $81-90 \%$; and 10, 91-100\%. IRS was calculated by multiplying the staining intensity score with the staining percentage score. Any disagreement in the IRS score between the two pathologists was resolved by discussion. The mean IRS score from 80 patients with CSCC was calculated to be $5.36 \pm 3.48$ and this was used as the cut-off value. Cases with an IRS score $\geq 5$ were included in the high ZIC1 expression group, and cases with an IRS score $<5$ were included in the low ZIC1 expression group.

Statistical analysis. Continuous variables were expressed as the mean \pm standard deviation and analyzed by using a 

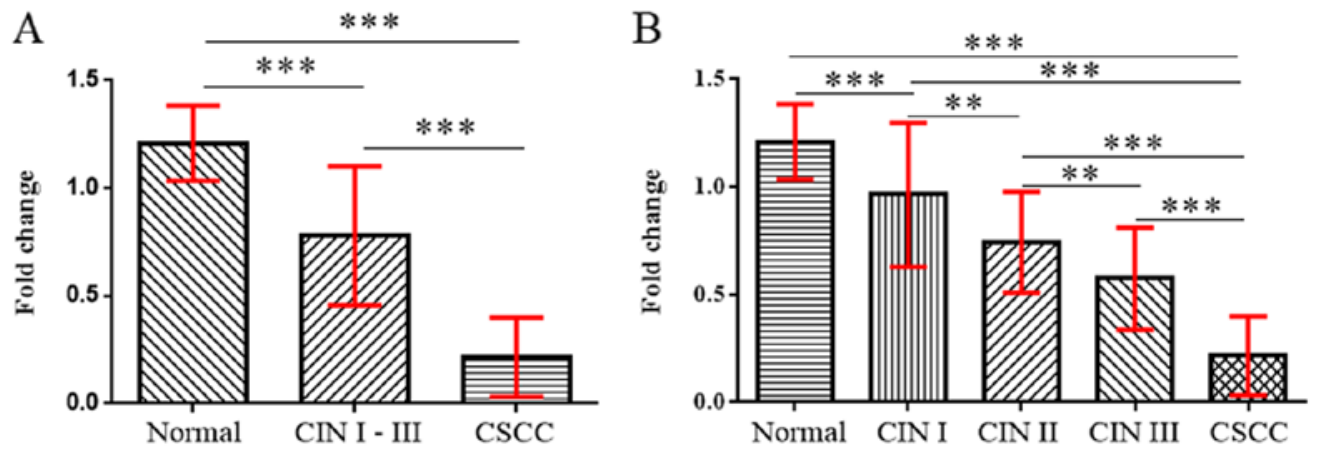

Figure 1. Expression of ZIC1 mRNA in biopsy tissues. (A) Expression of ZIC1 in normal tissues, CIN and CSCC samples determined by RT-qPCR. (B) Expression of ZIC1 in normal tissues, CIN I, II and III, and CSCC samples determined by RT-qPCR. ${ }^{* *} \mathrm{P}<0.01$; ${ }^{* * * *} \mathrm{P}<0.001$. ZIC1, zinc finger of the cerebellum 1; CSCC, cervical squamous cell carcinoma; CIN, cervical intraepithelial neoplasia; RT-qPCR, reverse transcription-quantitative PCR.

A

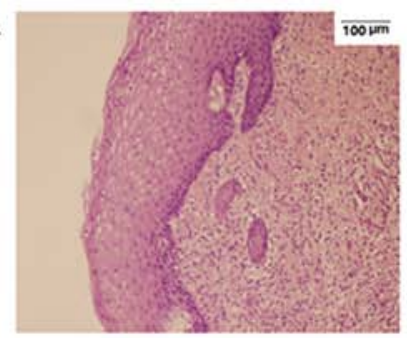

B

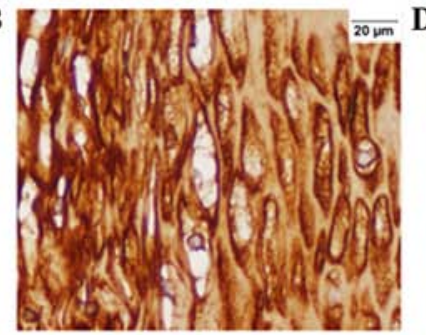

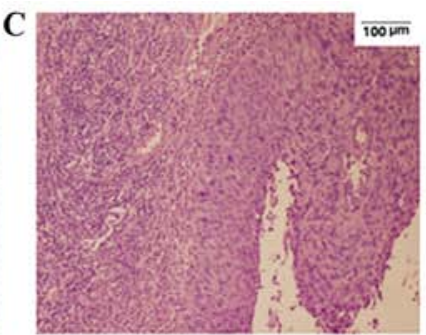

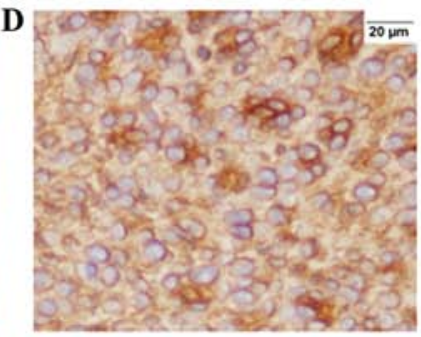

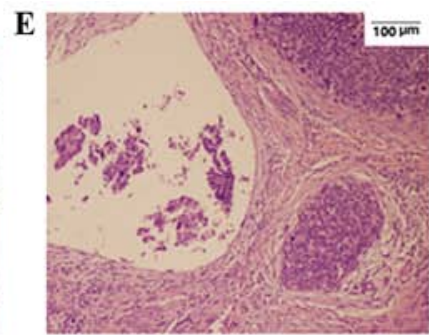
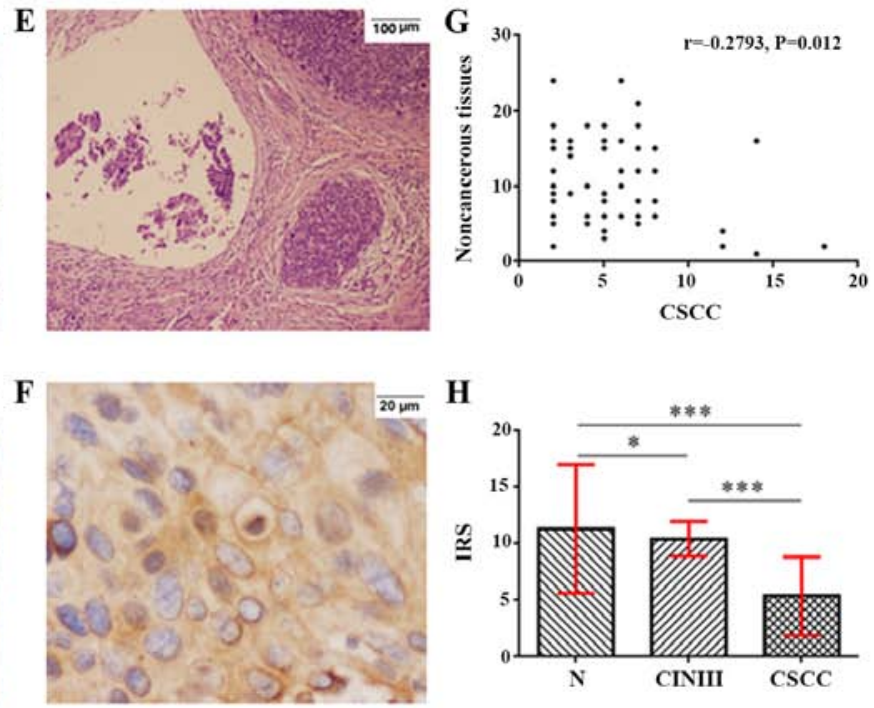

Figure 2. Expression of ZIC1 protein in CIN III samples, CSCC and adjacent noncancerous tissues. Hematoxylin and eosin staining of (A) noncancerous tissues, (C) CIN III and (E) CSCC tissues (magnification x100). Immunohistochemistry staining for ZIC1 protein (B) in noncancerous tissues, (D) CIN III and (F) CSCC tissues (magnification $\mathrm{x} 400$ ). (G) There was a negative correlation between ZIC1 expression in CSCC tissues and expression in adjacent noncancerous tissues. (H) Statistical comparison of IRS of ZIC1 staining in CSCC, noncancerous tissues and CIN III samples. ${ }^{*} \mathrm{P}<0.05$, ${ }^{* * *} \mathrm{P}<0.001$. CSCC, cervical squamous cell carcinoma; N, corresponding adjacent noncancerous tissues; CIN, cervical intraepithelial neoplasia; ZIC1, zinc finger of the cerebellum 1; IRS, immunoreactivity score.

one-way ANOVA with a post-hoc Least Significant Difference test. Spearman's rank correlation was performed to identify potential correlations in the IRS score between CSCC and the corresponding adjacent noncancerous tissues. A Pearson's $\chi^{2}$ test was used to analyze the association between ZIC1 expression and the clinicopathological characteristics of patients with CSCC. Cox regression analysis and Kaplan-Meier curves combined with a Log Rank test were used to analyze overall survival (OS) and disease-free survival (DFS). SPSS version 20.0 (IBM, Corp.) and GraphPad version 6.0 (GraphPad Software, Inc.) were used for statistical analyses. $\mathrm{P}<0.05$ was considered to indicate a statistically significant difference.

\section{Results}

ZIC1 mRNA expression levels in biopsy tissues. The mRNA expression levels of ZIC1 in CSCC, CIN and normal cervical tissues were determined using RT-qPCR (Fig. 1). The level of ZIC1 mRNA expression in CSCC was significantly lower compared with normal cervical tissues or in CIN I-III (P<0.001; Fig. 1). ZIC1 mRNA expression levels in CIN I-III were also significantly lower compared with normal cervical tissues ( $\mathrm{P}<0.001$; Fig. 1A). In addition, ZIC1 mRNA expression levels in CIN were associated with CIN grade, as lower expression was observed with increasing CIN grade $(\mathrm{P}<0.01$; Fig. 1B). ZIC1 expression in any CIN grade was significantly lower compared with normal cervical tissues $(\mathrm{P}<0.01$ or $\mathrm{P}<0.001$; Fig. 1A).

Expression of ZIC1 in CSCC and CIN III samples. Hematoxylin and eosin staining of noncancerous, CIN III and CSCC tissues are presented in Fig. 2A, C and E, respectively. ZIC1 was expressed in the cytoplasm of cells in noncancerous tissues (Fig. 2B), CIN III samples (Fig. 2D) and CSCC (Fig. 2F). A negative correlation was observed between the IRS of ZIC1 expression in CSCC with the corresponding adjacent noncancerous tissues ( $\mathrm{r}=-0.279 ; \mathrm{P}=0.012$; Fig. $2 \mathrm{G}$ ). The mean $\mathrm{ZIC1}$ IRS in CSCC was 5.36 \pm 3.48 , significantly lower compared 
Table I. Association between ZIC1 expression in cervical squamous cell carcinoma and clinicopathological variables of patients.

\begin{tabular}{|c|c|c|c|c|c|}
\hline \multirow[b]{2}{*}{ Variable } & \multirow[b]{2}{*}{$\mathrm{n}$} & \multicolumn{2}{|c|}{ ZIC1 expression } & \multirow[b]{2}{*}{$\chi^{2}$ value } & \multirow[b]{2}{*}{ P-value } \\
\hline & & $\mathrm{High}^{\mathrm{a}}, \mathrm{n}(\%)$ & $\mathrm{Low}^{\mathrm{b}}, \mathrm{n}(\%)$ & & \\
\hline Total & 80 & $46(100.00)$ & $34(100.00)$ & & \\
\hline Age, years & & & & 0.101 & 0.750 \\
\hline$\leq 53$ & 36 & $20(43.48)$ & $16(47.06)$ & & \\
\hline$>53$ & 44 & $26(56.52)$ & $18(52.94)$ & & \\
\hline Tumor size, $\mathrm{cm}$ & & & & 0.077 & 0.782 \\
\hline$\leq 2$ & 48 & $27(58.70)$ & $21(61.76)$ & & \\
\hline$>2$ & 32 & $19(41.30)$ & $13(38.24)$ & & \\
\hline Tumor grading & & & & 0.272 & 0.873 \\
\hline G1 & 22 & $12(26.09)$ & $10(29.41)$ & & \\
\hline $\mathrm{G} 2$ & 38 & $23(50.00)$ & $15(44.12)$ & & \\
\hline G3 & 20 & $11(23.91)$ & $9(26.47)$ & & \\
\hline FIGO stage & & & & 7.190 & 0.027 \\
\hline IA & 14 & $10(21.74)$ & $4(11.76)$ & & \\
\hline IB & 37 & $25(54.35)$ & $12(35.30)$ & & \\
\hline IIA & 29 & $11(23.91)$ & $18(52.94)$ & & \\
\hline Lymph node metastasis & & & & 14.403 & $<0.001$ \\
\hline No & 31 & $26(56.52)$ & $5(14.71)$ & & \\
\hline Yes & 49 & $20(43.48)$ & $29(85.29)$ & & \\
\hline Vascular invasion & & & & 0.812 & 0.368 \\
\hline No & 52 & $28(60.87)$ & $24(70.59)$ & & \\
\hline Yes & 28 & $18(39.13)$ & $10(29.41)$ & & \\
\hline
\end{tabular}

${ }^{a}$ High ZIC1 expression was defined as IRS $\geq 5$; ${ }^{b}$ low ZIC1 expression was defined as IRS <5. ZIC1, zinc finger of the cerebellum 1; IRS, immunoreactivity score; FIGO, International Federation of Gynecology and Obstetrics.

with that in the corresponding adjacent noncancerous tissues $(11.31 \pm 5.68 ; \mathrm{P}<0.001)$ and the CIN III samples $(10.42 \pm 1.54$; $\mathrm{P}<0.001$; Fig. $2 \mathrm{H}$ ). In addition, the mean IRS of ZIC1 in noncancerous tissues was significantly higher compared with CIN III samples ( $\mathrm{P}=0.014$; Fig. $2 \mathrm{H})$.

Association between ZIC1 expression and clinicopathological features in patients with CSCC. As the mean IRS of ZIC1 expression in CSCC was 5.36 \pm 3.48 , an IRS of 5 was used as the cut-off value. Cases with an IRS $\geq 5$ were included in the high ZIC1 expression group, and cases with IRS $<5$ were included in the low ZIC1 expression group. In Table I, high expression of ZIC1 was negatively associated with FIGO stage $(\mathrm{P}=0.027)$ and lymph node metastasis $(\mathrm{P}<0.001)$, but there were no significant associations with any of the other clinicopathological characteristics (age, tumor size, tumor grading and vascular invasion) between high and low ZIC1 expression $(\mathrm{P}>0.05)$.

OS. Kaplan-Meier analysis showed that the OS time of the low ZIC1 expression group (39.62 \pm 4.06 months) was significantly lower compared with the high ZIC1 expression group (51.26 \pm 2.44 months; $\mathrm{P}=0.019$; Fig. 3A). In univariate Cox regression analysis (Table $\mathrm{II}), \mathrm{ZIC1}(\mathrm{P}=0.027)$, tumor grading $(\mathrm{P}=0.020)$, lymph node metastasis $(\mathrm{P}=0.003)$ and FIGO stage $(\mathrm{P}<0.001)$ were associated with OS. In multivariate analysis,
ZIC1 expression (HR, 0.61; 95\% CI, 0.40-0.92; P=0.018), FIGO staging (HR, 3.55; 95\% CI, 2.35-5.37; P<0.001) and lymph node metastasis (HR, 2.50; 95\% CI, 1.62-3.86; $\mathrm{P}<0.001$ ) were determined to be independent prognostic factors. Of the 320 patients with CIN grade III, there were no deaths in the 5 years following LEEP treatment.

DFS. In Fig. 3B, the DFS time of the low ZIC1 expression group (33.88 \pm 3.77 months; $\mathrm{P}=0.002)$ was significantly lower compared with the high ZIC1 expression group (48.65 \pm 2.53 months). In Cox regression analysis (Table III), ZIC1 expression $(\mathrm{P}=0.003)$, tumor grading $(\mathrm{P}=0.022)$, FIGO stage $(\mathrm{P}<0.001)$ and lymph node metastasis $(\mathrm{P}=0.006)$ were four independent factors of DFS. Of the 320 patients with CIN grade III, there were no incidences of recurrence in the 5 years following LEEP treatment.

\section{Discussion}

In developing countries, cervical cancer is one of the most common types of cancer in females, which has significant repercussions for the medical and academic fields (1). Although the 9-valent HPV vaccine has the potential to prevent HPV infection and the occurrence of high-risk HPV cervical cancer, widespread use of the vaccine has not been adopted due to 
Table II. Prognostic value of ZIC1 expression and clinicopathological factors for overall survival of patients with cervical squamous cell carcinoma.

\begin{tabular}{|c|c|c|c|c|c|c|}
\hline \multirow[b]{2}{*}{ Variable } & \multicolumn{3}{|c|}{ Univariate analysis } & \multicolumn{3}{|c|}{ Multivariate analysis } \\
\hline & $\mathrm{HR}$ & $95 \% \mathrm{CI}$ & P-value & HR & $95 \% \mathrm{CI}$ & P-value \\
\hline ZIC1 expression, high vs. low & 0.42 & $0.20-0.91$ & 0.027 & 0.61 & $0.40-0.92$ & $0.018^{\mathrm{a}}$ \\
\hline Age, $>53$ vs. $\leq 53$ years & 1.07 & $0.80-1.44$ & 0.652 & & & \\
\hline Tumor size, $>2$ vs. $<2 \mathrm{~cm}$ & 1.33 & $0.84-2.11$ & 0.217 & & & \\
\hline Tumor grading, G3 vs. G1 and G2 & 1.27 & $1.04-1.56$ & 0.020 & - & - & - \\
\hline FIGO stage, IIA vs. I & 2.32 & $1.68-3.19$ & $<0.001$ & 3.55 & $2.35-5.37$ & $<0.001$ \\
\hline Lymph node metastasis, yes vs. no & 1.52 & $1.16-2.00$ & 0.003 & 2.50 & $1.62-3.86$ & $<0.001$ \\
\hline Vascular invasion, yes vs. no & 1.05 & $0.81-1.36$ & 0.704 & & & \\
\hline
\end{tabular}

ZIC1, zinc finger of the cerebellum 1; HR, hazard ratio; CI, confidence interval; FIGO, International Federation of Gynecology and Obstetrics; - , no results.

Table III. Prognostic value of ZIC1 expression and clinicopathological factors for disease-free survival of patients with cervical squamous cell carcinoma.

\begin{tabular}{|c|c|c|c|c|c|c|}
\hline \multirow[b]{2}{*}{ Variable } & \multicolumn{3}{|c|}{ Univariate analysis } & \multicolumn{3}{|c|}{ Multivariate analysis } \\
\hline & HR & $95 \% \mathrm{CI}$ & P-value & HR & $95 \% \mathrm{CI}$ & P-value \\
\hline ZIC1 expression, high vs. low & 0.41 & $0.22-0.76$ & 0.005 & 0.69 & $0.55-0.88$ & 0.003 \\
\hline Age, $>53$ vs. $\leq 53$ years & 1.38 & $0.89-2.13$ & 0.146 & & & \\
\hline Tumor size, $>2$ vs. $<2 \mathrm{~cm}$ & 1.46 & $0.81-2.64$ & 0.205 & & & \\
\hline Tumor grading, G3 vs. G1 and G2 & 1.86 & $1.25-2.75$ & 0.002 & 1.59 & $1.07-2.35$ & 0.022 \\
\hline FIGO stage, IIA vs. I & 2.02 & $1.36-2.99$ & $<0.001$ & 2.89 & $1.95-4.29$ & $<0.001$ \\
\hline Lymph node metastasis, yes vs. no & 1.68 & $1.13-2.49$ & 0.010 & 2.23 & $1.26-3.94$ & 0.006 \\
\hline Vascular invasion, yes vs. no & 1.12 & $0.73-1.73$ & 0.597 & & & \\
\hline
\end{tabular}

ZIC1, zinc finger of the cerebellum 1; HR, hazard ratio; CI, confidence interval; FIGO, International Federation of Gynecology and Obstetrics.
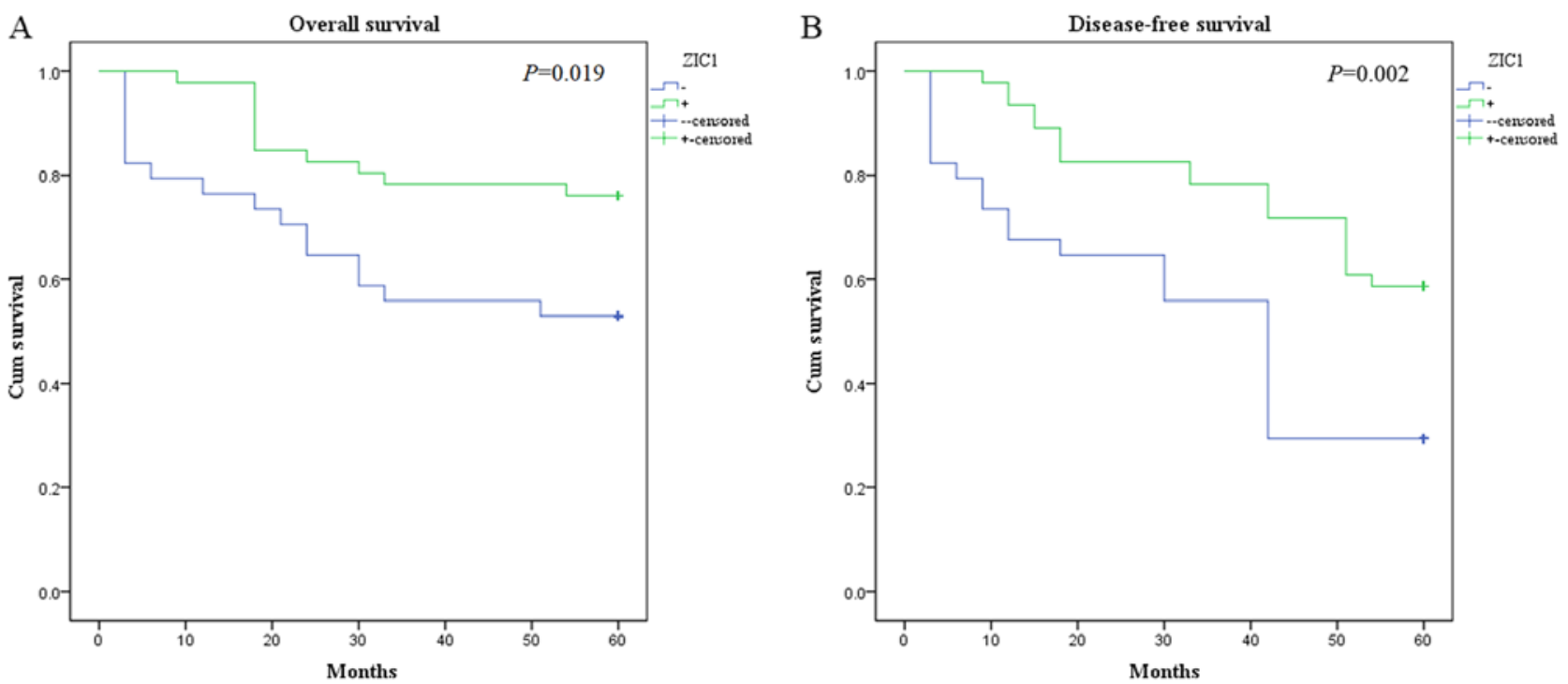

Figure 3. Kaplan-Meier survival curves of patients with cervical squamous cell carcinoma stratified by high or low expression of ZIC1. (A) Overall survival and (B) disease-free survival. Blue lines represent the low ZIC1 expression group and the green lines represent the high ZIC1 expression group. ZIC1, zinc finger of the cerebellum 1 . 
adverse side effects and economic burdens $(15,16)$. In addition, cervical cancer is still one of the leading causes of cancer-associated deaths in females, and it has a high rate of morbidity and mortality (1). Therefore, novel biomarkers are required to improve diagnosis and prognosis of patients with CSCC.

The ZIC family of proteins were named as such due to the high levels of expression of zinc finger proteins in cerebellar granule cells, and ZIC1 is essential for metabolic and physiological functions associated with the zinc finger proteins (4). Recent studies have shown that ZIC1 is a putative tumor suppressor gene, and its expression is closely associated with the occurrence and development in a variety of different types of tumors, such as ovarian cancer, thyroid cancer and breast cancer (17-20). Methylation of ZIC1 is frequently observed in patients with ovarian cancer, and its hypermethylation can contribute to cisplatin resistance in ovarian cancer cells (17). DNA methylation is significantly associated with carcinogenesis $(21,22)$. In addition, ZIC1 expression was found to be significantly reduced in malignant thyroid cells compared with that in normal thyroid cells, and was typically associated with methylation of the ZIC1 promoter (19). Ectopic expression of ZIC1 inhibited the growth of thyroid cancer cell lines (BCPAP, 8305C and C643 cells) by modulating the PI3K/Akt and MAPK signaling pathways and the FOXO3a transcription factor (19). In addition, $\mathrm{ZIC1}$ inhibited cell proliferation, reduced mitochondrial membrane potential and promoted apoptosis of breast cancer cells by inactivating the Akt/mTOR/P70S6K pathway, suppressing survivin expression, modulating the cell cycle and activating the mitochondrial apoptotic pathway (23). Additionally, previous studies revealed that expression of ZIC1 was upregulated in endometrial cancer compared with the corresponding normal tissue, and was positively correlated with age, disease stage, Tumor-Node-Metastasis stage and FIGO stage $(10,11)$. The findings of the current study revealed that the levels of ZIC1 mRNA in CSCC samples were significantly decreased compared to the levels in normal cervical tissues and CIN samples. In addition, ZIC1 expression in CIN samples was significantly lower compared with normal cervical tissues, and ZIC1 expression was found to be significantly reduced with increased CIN grade. Verlaat et al (6) showed that methylation of $\mathrm{ZIC1}$ was associated with the development and progression of $\mathrm{CC}$, which may explain the effect of downregulation of ZIC1 in CSCC and CIN samples. Furthermore, ZIC1 protein expression in 80 cases of CSCC were determined using IHC in the present study. Protein expression levels were significantly lower compared with the corresponding adjacent noncancerous tissues or CIN III samples.

ZIC1 expression is disordered in tumors, but not in normal tissues and its abnormal expression is associated with malignant biological behaviors $(8,9,11)$. In the present study, ZIC1 expression in CSCC was significantly lower compared with normal tissues and high expression of ZIC1 was negatively associated with FIGO stage and lymph node metastasis. In addition, the OS rate and DFS rate in patients with low ZIC1 expression were both significantly lower compared with patients with high ZIC1 expression, and ZIC1 expression was determined to be an independent prognostic marker for CSCC. Previous research revealed that ZIC1 was a novel indicator of prognosis in patients with invasive breast cancer and gastric cancer $(8,9)$. Therefore, in the present study, it was demonstrated that ZIC1 may additionally be a reliable biomarker for patients with CSCC. However, the limitation of the current study was that it was focused only on the prognostic value of $\mathrm{ZIC1}$ in cervical squamous cell carcinoma. Based on these findings, functional studies should be performed to ascertain the effect of ZIC1 expression both in vitro and in vivo in CSCC.

The present study showed that ZIC1 may be a novel indicator of clinicopathological features and prognosis in patients with CSCC. However, it should be noted that only patients classified with FIGO stage I-IIA were included in the present study, and patients who had undergone surgery or with a tumor classified at FIGO IIB-IV CSCC were excluded. Further research is required to confirm the clinical value of $\mathrm{ZIC1}$ expression in patients with FIGO stage IIB-III who received chemoradiotherapy prior to surgery.

In conclusion, ZIC1 expression in CSCC was significantly lower compared with normal cervical tissues and with CIN samples. In addition, ZIC1 expression in CIN was significantly lower compared with expression in normal cervical tissues, and was associated with CIN grade. High expression of ZIC1 was negatively associated with FIGO stage and lymph node metastasis, and it was associated with improved OS and DFS in patients with CSCC. Further research is required to confirm the clinical value of $\mathrm{ZIC1}$ in patients with CSCC and to understand the underlying mechanism of ZIC1 expression in CSCC.

\section{Acknowledgements}

Not applicable.

\section{Funding}

The present study was supported by the Kunshan Science and Technology Program of Social Development (grant no. KS1730).

\section{Availability of data and materials}

The datasets used and/or analyzed during the present study are available from the corresponding author on reasonable request.

\section{Authors' contributions}

XG and QL conceived and designed the study. XG, XKG and BHC performed the experiments and wrote the manuscript. $\mathrm{XG}$, XJG and FC analyzed the data. All authors read and approved the manuscript and agreed to be accountable for all aspects of the research in ensuring that the accuracy or integrity of any part of the work are appropriately investigated and resolved.

\section{Ethics approval and consent to participate}

The present study was approved by The Ethics Committee of Kunshan First People's Hospital (Jiangsu, China), and consent was obtained from each patient for the use of their tissues for research and publication.

\section{Patient consent for publication}

Not applicable. 


\section{Competing interests}

The authors declare that they have no competing interests.

\section{References}

1. Siegel RL, Miller KD and Jemal A: Cancer statistics, 2018. CA Cancer J Clin 68: 7-30, 2018

2. Robadi IA, Pharaon M and Ducatman BS: The importance of high-risk human papillomavirus types other than 16 and 18 in cervical neoplasia. Arch Pathol Lab Med 142: 693-695, 2018.

3. Solís JG and Briones-Torres TI: Prevalence of intraepithelial lesion in cervical screening cytology in a First-level Care Unit. Rev Med Inst Mex Seguro Soc 56: 167-172, 2018 (In Spanish; Abstract available in Spanish from the publisher).

4. Aruga J, Yokota N, Hashimoto M, Furuichi T, Fukuda M and Mikoshiba K: A novel zinc finger protein, zic, is involved in neurogenesis, especially in the cell lineage of cerebellar granule cells. J Neurochem 63: 1880-1890, 1994.

5. Aruga $J$ and Millen KJ: ZIC1 Function in normal cerebellar development and human developmental pathology. Adv Exp Med Biol 1046: 249-268, 2018.

6. Verlaat W, Snijders PJF, Novianti PW, Wilting SM, De Strooper LMA, Trooskens G, Vandersmissen J, Van Criekinge W, Wisman GBA, Meijer CJLM, et al: Genome-wide DNA methylation profiling reveals methylation markers associated with $3 \mathrm{q}$ gain for detection of cervical precancer and cancer. Clin Cancer Res 23: 3813-3822, 2017.

7. Gan L, Chen S, Zhong J, Wang X, Lam EK, Liu X, Zhang J, Zhou T, Yu J, Si J, et al: ZIC1 is downregulated through promoter hypermethylation, and functions as a tumor suppressor gene in colorectal cancer. PLoS One 6: e16916, 2011.

8. Han W, Zhang C, Gao XJ, Wang HB, Chen F, Cao F, Hu YW, Ma J, Gu X and Ding HZ: Clinicopathologic and prognostic significance of the zinc finger of the cerebellum family in invasive breast cancer. J Breast Cancer 21: 51-61, 2018.

9. Ma G, Dai W, Sang A, Yang X and Li Q: Roles of ZIC family genes in human gastric cancer. Int J Mol Med 38: 259-266, 2016

10. Wong YF, Cheung TH, Lo KW, Yim SF, Siu NS, Chan SC, Ho TW, Wong KW, Yu MY, Wang VW, et al: Identification of molecular markers and signaling pathway in endometrial cancer in Hong Kong Chinese women by genome-wide gene expression profiling. Oncogene 26: 1971-1982, 2007.

11. Gu X, Liu Q, Yang N, Shen JF, Zhang XG, Cao F and Ding HZ: Clinicopathological significance of increased ZIC1 expression in human endometrial cancer. J Huazhong Univ Sci Technolog Med Sci 35: 898-903, 2015.

12. Lim S, Cho K, Lee S, Lee K, Shin J, Chung D and Park C: Effect of number of retrieved lymph nodes on prognosis in FIGO stage IB-IIA cervical cancer patients treated with primary radical surgery. J Obstet Gynaecol Res 43: 211-219, 2017.
13. Ginocchio CC, Barth D and Zhang F: Comparison of the Third Wave Invader human papillomavirus (HPV) assay and the digene HPV hybrid capture 2 assay for detection of high-risk HPV DNA. J Clin Microbiol 46: 1641-1646, 2008.

14. Livak KJ and Schmittgen TD: Analysis of relative gene expression data using real-time quantitative PCR and the 2(-Delta Delta C(T)) method. Methods 25: 402-408, 2001.

15. Guevara A, Cabello R, Woelber L, Moreira ED Jr, Joura E, Reich O, Shields C, Ellison MC, Joshi A and Luxembourg A: Antibody persistence and evidence of immune memory at 5years following administration of the 9-valent HPV vaccine. Vaccine 35: 5050-5057, 2017.

16. Martínez-Lavín M and Amezcua-Guerra L: Serious adverse events after HPV vaccination: A critical review of randomized trials and post-marketing case series. Clin Rheumatol 36: 2169-2178, 2017.

17. Fang F, Munck J, Tang J, Taverna P, Wang Y, Miller DF, Pilrose J, Choy G, Azab M, Pawelczak KS, et al: The novel, small-molecule DNA methylation inhibitor SGI-110 as an ovarian cancer chemosensitizer. Clin Cancer Res 20: 6504-6516, 2014.

18. Du L, Qian X, Dai C, Wang L, Huang D, Wang S and Shen X: Screening the molecular targets of ovarian cancer based on bioinformatics analysis. Tumori 101: 384-389, 2015.

19. Qiang W, Zhao Y, Yang Q, Liu W, Guan H, Lv S, Ji M, Shi B and Hou P: ZIC1 is a putative tumor suppressor in thyroid cancer by modulating major signaling pathways and transcription factor FOXO3a. J Clin Endocrinol Metab 99: E1163-E1172, 2014.

20. Chen X, Lin Z, Xue M, Si J and Chen S: Zic1 promoter hypermethylation in plasma DNA is a potential biomarker for gastric cancer and intraepithelial neoplasia. PLoS One 10: e0133906, 2015.

21. van der Zee RP, Richel O, van Noesel CJM, Novianti PW, Ciocanea-Teodorescu I, van Splunter AP, Duin S, van den Berk GEL, Meijer CJLM, Quint WGV, et al: Host cell deoxyribonucleic acid methylation markers for the detection of high-grade anal intraepithelial neoplasia and anal cancer. Clin Infect Dis 68: 1110-1117, 2019.

22. Baribault C, Ehrlich KC, Ponnaluri VKC, Pradhan S, Lacey M and Ehrlich M: Developmentally linked human DNA hypermethylation is associated with down-modulation, repression, and upregulation of transcription. Epigenetics 13: 275-289, 2018.

23. Han W, Cao F, Gao XJ, Wang HB, Chen F, Cai SJ, Zhang C, $\mathrm{Hu}$ YW, Ma J, Gu X and Ding HZ: ZIC1 acts a tumor suppressor in breast cancer by targeting survivin. Int J Oncol 53: 937-948, 2018.

This work is licensed under a Creative Commons Attribution-NonCommercial-NoDerivatives 4.0 International (CC BY-NC-ND 4.0) License. 